\title{
Welcome to the International Journal of Prognostics and Health Management (Communication)
}

\author{
Neil H. W. Eklund ${ }^{1}$ \\ ${ }^{I}$ General Electric Global Research, One Research Circle, Niskayuna, NY 12309, USA \\ editor@ijPHM.org
}

\section{INTRODUCTION TO ijPHM}

The Prognostics and Health Management Society (PHM Society) is a non-profit organization dedicated to the advancement of PHM as an engineering discipline. One of the fundamental principles of the Society is to provide centralized, timely, free and unrestricted access to knowledge about PHM research and applications.

The flagship publication of the PHM Society is the open online journal, International Journal of Prognostics and Health Management (ijPHM), publishing multidisciplinary research on Prognostics, Diagnostics, and System Health Management. In contrast to most other technical organizations, PHM Society has adopted a Creative Commons license policy towards its archival journal which allows authors to retain copyright while allowing the Society to distribute their work broadly through modern media. Papers published in ijPHM are available for download to everyone, everywhere, without restriction, and at no cost.

The focus of ijPHM is to promote the exchange of innovative ideas and to advance PHM as a scientific discipline. The editorial team at ijPHM is dedicated to providing a very short publication cycle - papers are reviewed within eight weeks of initial submission, and articles are published as soon as an acceptably revised version is received. This pace is much faster than what is possible with traditional print media, allowing results from PHM research and application to be broadly disseminated while they are most relevant, promoting rapid progress in the field. However, this fast pace does not diminish the level of scientific rigor required for publication - each article will be subjected to peer review by at least three independent scientists with established track records in the field.

This is an open-access article distributed under the terms of the Creative Commons Attribution 3.0 United States License, which permits unrestricted use, distribution, and reproduction in any medium, provided the original author and source are credited.
Because ijPHM is an online publication, it is free of the limitations of print media. The length of regular journal articles is unrestricted, placing no arbitrary limit on the number of pages necessary to describe and interpret the work clearly in the context of other research. The appropriate use of color in figures and color photographs are encouraged. Raw or processed experimental data and video that elegantly illustrates specific, relevant scientific aspects of the paper may be attached to the publication. Papers may also include links to web sites, other publications, and to other sections of the same document. Looking ahead, we are also considering unconventional ideas such as "living articles" that can be revised and expanded over time, on-demand printing of customized volumes, and allowing public discussion threads for published papers.

\section{INFORMATION FOR AUTHORS}

ijPHM publishes scientific papers dealing with all aspects of prognostics, diagnostics, and system health management of complex engineered systems. We accept high quality articles focused on assessing the current status and predicting the future condition of an engineered component and/or system of components. Such articles may come from a variety of disciplines, including electrical, electronics, mechanical, civil, and chemical engineering, computer and materials science, reliability, test and measurement, artificial intelligence, physics, and economics. Contributions to ijPHM must report original research and will be subjected to review by referees at the discretion of the Editor. ijPHM considers only manuscripts that have not been published elsewhere (including at conferences), and that are not under consideration for publication or in press elsewhere. Moreover, it is the responsibility of the author to ensure that any data or information submitted complies with the export-control regulations of the author's home country, e.g., International Traffic in Arms Regulations (ITAR) in the United States.

Submitted 10/2009; published 10/2009. 
ijPHM publishes full-length regular papers, technical briefs, communications, and survey papers. Full-length regular papers should describe new and carefully confirmed findings. The experimental methods used, results obtained, analysis, and conclusions must be presented clearly and objectively, such that they might be replicated by another researcher. A full paper should be long enough to describe and interpret the work clearly, placing it in the context of other research.

Technical briefs typically describe a single result, experiment, or technique of general interest for which a short treatment is appropriate. Any experimental methods used, results obtained, analysis, and conclusions must be presented clearly and objectively, such that they might be replicated by another researcher. A technical brief should be long enough to describe and interpret the work clearly, although not necessarily in the context of other research.

Communications are a separate class of short manuscripts that are subject to an expedited review process. Appropriate items include (but are not limited to) rebuttals and/or counterexamples of previously published papers. A communication is suitable for highlighting the results of newly-completed projects or providing details of new models or hypotheses, innovative methods, techniques or apparatus. The style of main sections need not conform to that of full-length papers. Short communications are 2 to 4 printed pages in length. The Editors will review these submissions internally, and request outside review when appropriate.

Survey papers covering emerging research topics in PHM are also published, and unsolicited manuscripts of a tutorial or review nature are welcome. However, prospective authors of survey papers should contact the Editor-in-Chief in advance in order to assess the possible appeal of the topic for publication in ijPHM. Papers describing specific current applications are encouraged, provided that the designs represent the best current practice, detailed characteristics and performance are included, the application is discussed in the context of the state-of-the-art in the field, and it is of general interest.

Prospective authors should note that ijPHM is focused on promoting the exchange of innovative ideas and advancing PHM as a scientific discipline. Poorly documented papers or papers using "proprietary" techniques may be rejected without a comprehensive review. Moreover, ijPHM should not be seen as advertising space! Excessive "branding" within a paper is also cause for rejection; e.g., "The CompanyBrand ${ }^{\mathrm{TM}}$ team used the magical CompanyBrand $^{\mathrm{TM}}$ preprocessing technique to prepare the data to extract the amazing CompanyBrand ${ }^{\mathrm{TM}}$ proprietary features (which we can't tell you about)." Generally, no specific mention of a particular business (except where necessary to specify a piece of equipment) is permitted beyond the author's affiliation listed beneath their name, or sponsoring organizations given credit in the acknowledgements section.

ijPHM provides a very short publication cycle. Papers are reviewed initially to screen for obvious fatal defects - e.g., unacceptable English usage, excessive branding, clearly insufficient context (cited literature), etc. Acceptable papers are then sent to one of the members of the Editorial Board to be assigned reviewers. All full-length papers are reviewed by a minimum of three qualified reviewers. Specific peer-review feedback is provided to the author within eight weeks of initial submission. If the paper is approved for publication, necessary revisions should be made and submitted within four weeks.

\section{CALL FOR PAPERS}

The Prognostics and Health Management Society invites you to submit scientific papers of the highest quality dealing with all aspects of prognostics, diagnostics, and system health management of complex engineered systems to ijPHM. Areas of interest include but are not limited to:

- PHM system design and engineering

- Physics of failure

- Software health management

- Structural health management

- PHM for electronics

- Health management for renewable energies

- Diagnosis methods

- Data-driven prognostics

- Model-based prognostics

- Standards and methodologies

- Fault-adaptive controls

- Technology maturation

- Return-on-investment analysis

- Deployed applications and success stories

We welcome both papers that focus on fundamental research and application-oriented papers from diverse application areas such as energy, aerospace, transportation, automotive, and industrial automation. 